\title{
ADOLESCENTES COM DIABETES MELLITUS TIPO 1: ESTRESSE, COPING E ADESÃO AO TRATAMENTO
}

\author{
Vanessa Marques Gibran Victório \\ Doutora em Psicologia como Ciência e Profissão pela \\ Pontifícia Universidade Católica de Campinas (PUC- \\ -Campinas), Brasil.
}

\section{André Luiz Monezi Andrade}

Pós-Doutor em Psicobiologia pela Universidade Federal de São Paulo e em Psicologia pela Pontifícia Universidade Católica de Campinas (PUC-Campinas). Doutora em Psicologia pela Pontifícia Universidade Católica de Campinas (PUC -Campinas) Brasil. Docente dedo Curso de Psicologia da Universidade de Sorocaba (UNISO), Brasil.

\section{Andressa Melina Becker da Silva}

Doutora em Psicologia pela Pontifícia Universidade Católica de Campinas (PUC -Campinas) Brasil. Docente dedo Curso de Psicologia da Universidade de Sorocaba (UNISO), Brasil.

\section{Wagner de Lara Machado}

Doutor em Psicologia pela Universidade Federal do Rio Grande do Sul (UFRGS). Docente orientador do Programa de Pós-Graduação em Psicologia da Pontifícia Universidade Católica do Rio Grande do Sul (PUC-RS), Brasil.

\section{Sônia Regina Fiorim Enumo}

Doutora em Psicologia Experimental pela Universidade de São Paulo (USP). Docente orientadora do Programa de Pós-Graduação em Psicologia e do Programa em Ciências da Saúde da Pontifícia Universidade Católica de Campinas (PUC-Campinas), Brasil.
Autor correspondente

André Luiz Monezi Andrade

andre.andrade@puc-campinas.edu.br
RESUMO: Este estudo verificou as relações entre estressores do diabetes mellitus tipo 1 e seu enfrentamento e os comportamentos de autocuidados em 10 adolescentes, que responderam os seguintes instrumentos: Questionário Sociodemográfico, Summary of Diabetes Self-Care Activities Questionnaire, o Questionnaire on Stress in Patientes with Diabetes-Revised, Adolescent/Child's Self-Report Responses to Stress-Diabetes, todos com validação linguística, e a Escala de Coping do Diabetes para Adolescentes, baseada na Motivational Theory of Coping. Observou-se baixa adesão aos autocuidados. São principais estressores a hipoglicemia, o regime terapêutico e as queixas somáticas, os quais ameaçam a necessidade psicológica básica de Competência e geram raiva. Os adolescentes lidam com o regime terapêutico de forma mal adaptativa, diminuindo a adesão à atividade física. Estressores relacionados à ansiedade/depressão apresentaram maior número de correlações, especialmente com respostas de estresse involuntárias e de desengajamento. Essa análise das variáveis motivacionais do processo de adesão ao tratamento fornece dados prescritivos para intervenções com essa população.

PALAVRAS-CHAVE: Estresse psicológico; Enfrentamento; Cooperação e Adesão ao Tratamento; Diabetes mellitus Tipo 1; Adolescente.

\section{ADOLESCENTS WITH DIABETES MELLITUS 1: STRESS, COPING AND ADHESION TO TREATMENT}

\begin{abstract}
This study analyses the relationship between stress causes from diabetes mellitus I, coping and self-care behavior in 10 adolescents who answered the Sociodemographic Questionnaire, Summary of Diabetes Self-Care Activities Questionnaire, Questionnaire on Stress in Patients with Diabetes-Revised, Adolescent/Child's Self-Report Responses to Stress-Diabetes, with linguistic validation, and Coping Diabetes Scale for Adolescents, based on the Motivational Theory of Coping. Low self-caring adhesion was detected. Main stressors are hypoglycemia, therapy regime and body complaints, all of which threat the psychological basic need of competence and produced anger. Adolescents cope inadequately with the therapy regime and lessen adhesion to physical activities. Stress causes related to anxiety/depression had the greatest number of correlations, particularly involuntary responses to stress and disengagement. Current analysis of motivational variables of adhesion to treatment provides prescriptive data for interventions with the population concerned.
\end{abstract}

KEY WORDS: Stress, Psychological; Coping; Treatment Adherence and Compliance; Diabetes mellitus I; Adolescent. 


\section{INTRODUÇÃO}

A Organização Mundial da Saúde (OMS) estima que a incidência global da diabetes cresceu quase $62 \%$, entre 2006 e $2016^{1}$. Neste mesmo relatório, o Brasil foi o quarto colocado no ranking global de portadores do diabetes mellitus (DM), com mais de 16 milhões de brasileiros. Assim, a DM é hoje considerada mundialmente uma doença epidêmica e um dos maiores desafios para o século 21, segundo a Sociedade Brasileira de Diabetes².

A maioria das doenças crônicas, como o diabetes mellitus tipo 1 (DM1), que também afeta os adolescentes, é caracterizada por uma fase aguda próxima do diagnóstico da doença, seguido por estresse prolongado associado ao tratamento contínuo 3 . Além disso, existem evidências de que as condições crônicas podem gerar maior estresse psicológico e físico, influenciando diretamente no enfrentamento da doença ${ }^{4}$. Alguns autores observaram que adolescentes com DM1 têm mais dificuldades para manter suas atividades, como estudar e participar de eventos sociais, pois estes normalmente envolvem consumo de alimentos que não podem fazer parte de sua dieta ${ }^{5-6}$.

Essas condições dificultam a adesão ao tratamento do DM1 por esses adolescentes, mas o suporte social pode atuar como fator de proteção para o controle glicêmico ${ }^{7}$. Nesse sentido, comportamentos de autocuidado, tais como a frequência de monitorização da glicemia e a quantidade de injeções de insulina, podem ser influenciados por estressores sociais e econômicos ${ }^{8}$. Portanto, lidar de forma adaptativa com os estressores da doença e das condições de vida pelos adolescentes pode favorecer a adesão ao tratamento, com consequências para o controle glicêmico e para a qualidade de vida desses indivíduos.

Dessa forma, o estudo do coping pode contribuir nesse processo, pois ele é um dos principais mediadores e moderadores entre os eventos estressantes e o bem-estar psicológico, social e físico ${ }^{9-11}$. Segundo alguns autores $^{8-10}$, um padrão de desengajamento composto por estratégias de enfrentamento (EE) de esquiva e negação está associado a uma pior qualidade de vida e a depressão, mas não ao descontrole glicêmico em pacientes com DM1. Embora o descontrole glicêmico esteja associado a pensamentos mágicos ou fantasiosos, ele é mediado pelo suporte ao automonitoramento do adolescente ${ }^{8}$. Ou seja, adolescentes que conseguem regular sua emoção, pensamentos e comportamentos, por meio da autopercepção, apresentam com menor frequência e intensidade pensamentos mágicos e fantasiosos, o que pode levar a um maior controle glicêmico. Nesse sentido, incentivar sua autonomia e apresentar EE voltadas à adaptação de situações, como a aceitação e a distração (engajamento por coping de controle secundário), e procurar lidar diretamente com o estressor ou com a emoção gerada, por meio de solução de problema ou regulação emocional (engajamento por coping de controle primário) são melhores preditores da qualidade de vida ${ }^{8,10}$.

Nesta pesquisa, adotou-se uma perspectiva desenvolvimental e de autorregulação do coping, com base na proposição de Compas e colaboradores ${ }^{4,12}$ e na Motivational Theory of Coping/Teoria Motivacional do Coping (TMC) ${ }^{13-16}$. A TMC considera como estressor qualquer evento que ameace ou desafie as necessidades psicológicas básicas (NPB) de relacionamento ou pertença, de competência e de autonomia. A TMC propõe um sistema hierárquico multidimensional, com categorias de baixa ordem, compostas pelas instâncias ou respostas de coping, que são agrupados de acordo com sua função em EE. Estas, por sua vez, são classificadas de acordo com sua função no processo adaptativo em 12 famílias de coping, relacionadas à percepção de ameaça ou desafio a três NPB: 1) Necessidade de Relacionamento: Autoconfiança e Busca de Suporte (percepção do estressor como desafio); Delegação e Isolamento Social (percepção do estressor como uma ameaça); 2) Necessidade de Competência: Solução de Problemas e Busca de Informação (desafio); Desamparo e Fuga (ameaça); 3) Necessidade de Autonomia: Acomodação e Negociação (desafio); Submissão e Oposição (ameaça). Assim, diferentes EE podem ser apresentadas caso o estressor seja avaliado ou percebido como uma ameaça ou um desafio ${ }^{13}$.

Um contexto estruturado na vida do adolescente promove o engajamento ${ }^{4}$, favorecendo o tratamento. Essas condições são formadas com base na autopercepção de competência ou de autoeficácia para lidar com os estressores da doença ${ }^{10}$. Assim, essas relações sugerem que lidar com os estressores pode ter implicações importantes para a saúde dos adolescentes e, consequentemente, influenciam o processo de adesão aos comportamentos 
de autocuidado, imprescindíveis para um bom ajustamento glicêmico e uma melhor qualidade de vida desses jovens ${ }^{10}$. Nesse sentido, compreender essas relações e as EE pode ser importante para a promoção de comportamentos mais adaptativos desses adolescentes. Com essa perspectiva, foi proposto este estudo visando identificar e analisar as relações entre os estressores, as estratégias de enfrentamento de adolescentes com DM1, bem como sua adesão ao tratamento da doença.

\section{METODOLOGIA}

Este estudo foi realizado com uma amostra de conveniência de 10 adolescentes diagnosticados com DM1, na faixa etária de 12 a 15 anos. Dois adolescentes frequentavam uma Associação de Diabetes Juvenil (ADJ) de uma cidade do interior do Estado de São Paulo e os demais foram indicados por médicos da cidade, sendo feito o contato em suas residências. Mediante a concordância do adolescente e de seu responsável, os instrumentos foram aplicados em um único encontro, por escolha dos adolescentes e pela dificuldade no comparecimento para uma nova sessão. Eles demoraram, em média, 50 minutos para responder os instrumentos. Após a coleta de dados, foi oferecida uma devolutiva individual para o adolescente e seu responsável, explicando a necessidade dos comportamentos de autocuidado no processo de adesão ao tratamento. Também foram abordados e explicados sobre os principais estressores ligados ao diabetes e possíveis formas de lidar com eles. Este estudo foi aprovado pelo Comitê de Ética em Pesquisa da Universidade (Parecer $\mathrm{n}^{0}$ 945.500). Após a aprovação, foram respondidos pelos participantes os seguintes instrumentos:

Questionário Sociodemográfico - composto por oito itens: idade, gênero, escolaridade, composição familiar, tempo de diagnóstico da doença, religião e ocupação. O nível socioeconômico foi avaliado pelos Critérios de Classificação Econômica Brasil ${ }^{17}$. Para o perfil da amostra, foram utilizadas as frequências das variáveis categóricas (sexo, idade, escolaridade, nível socioeconômico, uso ou não da bomba de insulina e itens das escalas), a partir do Teste de Qui-Quadrado, com significância de 5\%.

Summary of Diabetes Self-Care Activities Ques- tionnaire (SDSCA)/Questionário de Atividades de Autocuidado com o Diabetes $(\mathrm{QAD})^{14}$ - traduzido e adaptado ${ }^{19}$ para avaliar o regime de adesão ao tratamento do diabetes a partir de 18 questões, agrupadas em seis dimensões do autocuidado, com bom coeficiente de Cronba$c h$ para esta amostra $(\alpha=0,69): 1)$ Alimentação geral e específica; 2) Atividade física; 3) Uso da medicação; 4) Monitoramento da glicemia; 5) Cuidado com os pés; e 6) Tabagismo. A avaliação da adesão geral às práticas de autocuidado foi realizada a partir da somatória nas 15 primeiras perguntas do $\mathrm{QAD}$. Aqueles adolescentes com pontuação igual ou mais a 75 pontos foram considerados de "alta adesão" (com controle da doença) e aqueles com pontuação menor que 74 foram considerados de "baixa adesão" (sem controle).

Questionnaire on Stress in Patients with Diabetes-Revised/Questionário de Estresse Face a Diabetes-R $(\mathrm{QSD}-\mathrm{R})^{20}$ - com validação portuguesa ${ }^{21} \mathrm{e}$ uso autorizado para avaliar os níveis de estresse em pacientes diabéticos. O QSD-R é composto por 40 itens respondidos em escala de seis pontos, agrupados em seis subescalas e apresentando alto índice de confiabilidade nesta amostra ( $\alpha=$ 0,79): a) Queixas somáticas; b) Estresse em contextos sociais e escolares; c) Ansiedade/depressão; d) Estresse face às hipoglicemias; e) Estresse face ao médico; f) Estresse face ao tratamento. Valores mais elevados são indicadores de níveis de estresse mais altos.

Adolescent/Child's Self-Report Responses to $\underline{\text { Stress }}$ (RSQ-Pediatric Diabetes-D-Ped) ${ }^{22}$ - com validação linguística autorizada, este instrumento é um dos poucos específicos para essa população ${ }^{10}$. Na versão utilizada, o RSQ-D-Ped avalia a reatividade ao estresse e ao coping dos estressores do diabetes em cinco fatores: a) Engajamento por Coping de Controle Primário (9 itens), relativo a ações que visam a alterar a situação estressora ou da emoção por ela eliciada; b) Engajamento por Coping de Controle Secundário, com 12 itens, relativos aos esforços de adaptação à situação; e c) Desengajamento ( 9 itens), compostos por respostas voluntárias ao estresse; d) Engajamento Involuntário (9 itens); e) Desengajamento Involuntário (12 itens), relacionado à reatividade ao estresse. O RSQ é dividido em duas partes. Há uma escala de quatro pontos $(0=$ nunca; $1=$ às vezes; $2=$ quase sempre $; 3=$ sempre), com 10 itens, para avaliar o quanto 
os estressores do diabetes perturbam, considerando os últimos seis meses (somando de 0 a 30 pontos, sendo que escores mais altos indicam maiores níveis de estresse percebido). A seguir, o adolescente avalia o quanto acredita ter controle sobre esses estressores. Na segunda parte, responde a uma escala de quatro pontos $(1=$ nada até $4=$ muito), com 57 itens relacionados à forma como ele lida com esses estressores. Alguns desses itens têm respostas alternativas para assinalar. É gerada uma média da pontuação bruta e uma pontuação proporcional de cada fator em relação ao total das 57 respostas de coping e de reatividade ao estresse.

Escala de Coping do Diabetes para Adolescentes (COPE-DIA) - baseada e adaptada do instrumento para avaliação do coping segundo a TMC ${ }^{23}$, especialmente para este estudo. É uma escala do tipo likert de cinco pontos $(1=$ nem um pouco a $5=$ totalmente $)$, com 21 itens, para seis tipos de medidas: reação emocional; avaliação de ameaça; avaliação de desafio; orientação motivacional; identificação com o personagem; e a frequência das 12 famílias de coping, respondidos para cada uma de três situações estressantes, descritas em um pequeno texto ilustrado: S1-Estressores sociais (dificuldade para expor a doença), S2-Adesão ao tratamento (dieta, atividades físicas, monitoramento da glicemia) e S3-Relacionamento com os pais (controle parental da alimentação). Seis EE (Autoconfiança, Busca de Suporte Social, Solução de Problemas, Busca de Informações, Aceitação e Negociação) têm desfechos positivos no médio e longo prazo em termos desenvolvimentais e de saúde, e geram um escore médio de Coping Adaptativo; as outras seis EE (Delegação, Isolamento Social, Desamparo, Esquiva, Submissão e Oposição) geram um escore médio de Coping Mal Adaptativo. Obteve-se uma alta consistência interna para a escala no total, nas três situações, e para as EE (exceção para Autoconfiança), com valores de alfa de Cronbach acima de 0,70 .

As variáveis contínuas foram analisadas a partir da frequência absoluta, média, desvio padrão e a mediana a partir de valores mínimos e máximos. Os dados foram analisados pelo Teste U de Mann-Whitney. Para analisar a relação entre os escores das escalas foi utilizado o coeficiente de correlação de Sperman, devido à ausência de distribuição normal das variáveis e o tamanho reduzido da amostra. O nível de significância em todas as análises foi de $5 \%$.

\section{RESULTADOS}

A amostra foi composta por 10 adolescentes, com idade entre 12 e 15 anos $\left(M_{\text {idade }}=13,8 ; \pm 1,22\right)$, sendo cinco de cada sexo, com diagnóstico de DM1. Faziam uso de bomba de insulina 4 adolescentes. Sete adolescentes cursavam o Ensino Fundamental e três o Ensino Médio. Para 9 dos adolescentes, as mães eram responsáveis e um era cuidado pela avó. O estado civil predominante dos pais foi o casado $(n=8)$. Metade dos cuidadores familiares $(n=5)$ tinha Ensino Médio incompleto e os demais estudaram até o Ensino Fundamental. As famílias tinham um nível socioeconômico médio $(n=6)$ ou médio-alto $(n=3)$.

Considerando o tempo médio de cinco dias para avaliar uma boa adesão, os comportamentos de autocuidados com maior adesão, segundo o QAD, foram: "tomar medicamentos do diabetes conforme recomendado" $\left(M_{\text {dias }}=7,30 ; \pm 2,21\right)$, "tomar injeções de insulina conforme recomendado" $\left(M_{\text {dias }}=6,50 ; \pm 2,55\right)$ e "avaliar o açúcar no sangue" $\left(M_{\text {dias }}=6,60 ; \pm 2,72\right)$. O padrão alimentar mostrou um consumo mediano de doces por semana $\left(M_{\text {dias }}=4,30 ; \pm 2,83\right)$ e baixo consumo de porções de frutas e/ou vegetais $\left(M_{\text {dias }}=3,10 ; \pm 1,91\right)$. A maioria dos adolescentes relatou fazer de forma moderada a atividade física e o cuidado com os pés. Nenhum deles relatou ser fumante.

São estressores para esta amostra, de acordo com o QSD-R, o Estresse Face à Hipoglicemia ("Médicos diferentes dão-me informações diferentes em relação à doença"; "Sinto-me culpado quando não cumpro a dieta", por exemplo), seguido dos estressores do Regime Terapêutico ("Às vezes, penso que vou ter problemas no futuro"; "Não posso comer tanto como me apetecia", por exemplo) e relacionados às Queixas Somáticas ("Fico irritado e nervoso quando estou com hiperglicemia (valores altos)", por exemplo). No RSQ-D-Ped, cinco estressores apresentaram frequência maior ou igual a 50\%: "Pais lembrando constantemente para ter mais cuidado" (70\%), "Lidar com os cuidados do diabetes" (60\%), "Sentimento 
de culpa ou chateação por causa dos 'resultados ruins"' (60\%), "Dúvidas sobre contar às pessoas sobre o diabetes e como fazê-lo" (50\%) e "Ver minha família preocupando-se comigo" (50\%). O escore médio de estresse da amostra ficou abaixo da média de 15 pontos $(M=13,6$; \pm 5,62). Metade desses adolescentes relatou ter somente "um pouco" de controle sobre esses estressores listados no RSQ-D-Ped, enquanto a outra metade da amostra se avaliou como tendo "algum" controle. Nesses dois instrumentos que avaliaram os estressores do DM1, predominaram os cuidados com a saúde e o sentimento de culpa pelo não cumprimento das orientações médicas.

Os adolescentes respondem aos estressores do diabetes principalmente por respostas involuntárias, de acordo com o RSQ-D-Ped, com maior média em Engajamento Involuntário, com destaque para a Excitação Emocional e a Ruminação, além do Desengajamento Involuntário, com maior média para as respostas de Paralisação e Interferência Cognitiva. Entretanto, apresentaram também respostas mais adaptativas, como o Engajamento de Controle Secundário, incluindo Aceitação, Pensamento Positivo e Distração. O Pensamento Fantasioso foi a EE com maior frequência, e está relacionado ao Desengajamento (Tabela 1).

Tabela 1. Médias de respostas involuntárias ao estresse e coping do diabetes mellitus tipo 1 em adolescentes ( $\mathrm{N}$ $=10)$

\begin{tabular}{l|c|c|c|c}
\hline $\begin{array}{l}\text { Categorias de respostas ao } \\
\text { estresse (RSQ-D-Ped) }\end{array}$ & \multicolumn{2}{|c|}{ Média } & \multicolumn{2}{c}{$\begin{array}{c}\text { Média } \\
\text { Proporcional }\end{array}$} \\
\hline $\begin{array}{l}\text { Respostas voluntárias } \\
\text { Engajamento por coping de } \\
\text { controle primário }\end{array}$ & $\mathbf{M}$ & $\mathbf{D P}$ & $\mathbf{M p r}$ & $\mathbf{D P}$ \\
\hline Resolução de problemas & 7,1 & 2,47 & 0,17 & 0,02 \\
\hline Regulação emocional & 6,9 & 1,45 & 0,06 & 0,01 \\
\hline Expressão emocional & 7,2 & 2,1 & 0,06 & 0,01 \\
\hline $\begin{array}{l}\text { Engajamento por coping de } \\
\text { controle secundário }\end{array}$ & 27,8 & 6,2 & 0,23 & 0,05 \\
\hline Pensamento positivo & 7,5 & 2,42 & 0,06 & 0,02 \\
\hline Reestruturação cognitiva & 5,8 & 1,32 & 0,05 & 0,02 \\
\hline Aceitação & 7,5 & 1,72 & 0,06 & 0,02 \\
\hline Distração & 7,0 & 2,58 & 0,06 & 0,01 \\
\hline Desengajamento & 19,7 & 4,81 & 0,16 & 0,03 \\
\hline
\end{tabular}

\begin{tabular}{l|c|c|c|c|}
\hline $\begin{array}{c}\text { Categorias de respostas ao } \\
\text { estresse (RSQ-D-Ped) }\end{array}$ & \multicolumn{2}{|c|}{ Média } & \multicolumn{2}{c}{$\begin{array}{c}\text { Média } \\
\text { Proporcional }\end{array}$} \\
\hline Respostas voluntárias & $\boldsymbol{M}$ & $\mathbf{D P}$ & $\mathbf{M p r}$ & $\mathbf{D P}$ \\
\hline Evitação & 5,6 & 1,65 & 0,04 & 0,01 \\
\hline Negação & 5,8 & 2,2 & 0,05 & 0,02 \\
\hline Pensamento fantasioso & 8,3 & 2,16 & 0,07 & 0,01 \\
\hline Respostas involuntárias & & & & \\
\hline Engajamento involuntário & 33,4 & 9,73 & 0,26 & 0,04 \\
\hline Ruminação & 7,6 & 2,88 & 0,06 & 0,01 \\
\hline Pensamentos intrusivos & 6,0 & 2,45 & 0,05 & 0,01 \\
\hline Excitação fisiológica & 6,0 & 1,70 & 0,04 & 0,01 \\
\hline Excitação emocional & 8,0 & 2,98 & 0,06 & 0,02 \\
\hline Ação involuntária (impulsiva) & 5,8 & 1,32 & 0,05 & 0,01 \\
\hline $\begin{array}{l}\text { Desengajamento involun- } \\
\text { tário }\end{array}$ & 22,3 & 5,83 & 0,18 & 0,02 \\
\hline Entorpecimento emocional & 4,8 & 1,23 & 0,04 & 0,01 \\
\hline Interferência cognitiva & 5,9 & 1,97 & 0,05 & 0,01 \\
\hline Paralisação & 6,0 & 2,36 & 0,05 & 0,01 \\
\hline Fuga & 5,6 & 1,71 & 0,04 & 0,01 \\
\hline Nota. (a) Valory & & & & \\
\hline
\end{tabular}

Nota. (a) Valores ponderados, pois cada fator tem um número diferente de categorias e cada categoria tem três itens, respondidos em uma escala: $1=$ nunca; 2 = às vezes; 3 = quase sempre; 4 = sempre; Coping de controle primário: ações que visam a alterar a situação estressora ou da emoção por ela eliciada; Coping de controle secundário: respostas de adaptação ao problema; Reestruturação cognitiva: processo de reconceitualização de uma ideia ou atitude, vendo-a de uma perspectiva diferente; Ruminação: padrão de pensamentos persistentes, com foco em desfechos e emoções negativos; Pensamento intrusivos: eventos mentais que interrompem o curso da ação, a despeito dos esforços persistentes para evitá-los; Interferência cognitiva: bloqueio da aprendizagem ou memória pelo aprendizado ou recordação de outro material conflitante.

Nas questões do RSQ-D-Ped com alternativas de resposta, percebe-se que o estressor foi visto como um desafio pelos adolescentes, pois estes relataram ter um enfrentamento adaptativo, tendo em vista que indicaram, como suporte para a expressão e a regulação emocional, e para a solução de problemas, os pais (70\%), Deus (40\%), os irmãos (40\%) e os amigos (30\%). As principais formas de expressão emocional foram: "Ouvir música" (70\%), "Chorar" (40\%) e "Desenhar/pintar" (30\%). As reações corporais relacionadas à excitação fisiológica descritas pelos adolescentes foram: "Minha respiração acelera" (50\%) e "Meu coração dispara" (40\%). Já em relação à $e x$ citação emocional, os problemas com o diabetes geram 
"preocupação/ansiedade" (60\%), tristeza e medo (40\% cada); neste caso, o estressor sendo percebido como uma ameaça às suas NPB. As atividades mais frequentes como EE de distração foram: "Assistir televisão" (70\%), "Ouvir música" (60\%) e "Jogar videogame" (50\%). Por fim, os comportamentos de coping mais relatados para a regulação emocional foram: "Ouvir música" (90\%), "Descansar" (60\%) e "Fazer orações" (50\%).

A aplicação do COPE-DIA indicou que a reação emocional de raiva foi a mais frequente nas três situações de estresse (S1-Estressores sociais, S2-Adesão ao tratamento e S3-Relacionamento com os pais) e a reação de medo foi a menos frequente, o que indica que o estressor é percebido como uma ameaça às suas NPB, levando a um coping mal adaptativo. Mesmo apresentando EE relativas às famílias de coping menos adaptativas, como Isolamento Social (66,6\%) e Fuga (60\%), mostrando que o estressor é entendido como ameaça à NPB de Relacionamento e de Competência respectivamente, houve predominância de famílias de coping adaptativas em duas situações (S1-Estressores sociais e S2-Adesão), com a Busca de Suporte (56,6\%), relacionada à percepção de desafio à NPB de Relacionamento, além da Solução de Problema $(53,3 \%)$ e da Busca de Informação (46,6\%), ambas relativas à percepção de desafio à NPB de Competência. Na S3-Relacionamento com os pais, predominou a família de coping de Isolamento (ameaça à NPB de Relacionamento).

Associações entre estressores do diabetes e seu enfrentamento, e os comportamentos de autocuidado

A subescala de estresse do QSD-R com maior quantidade de correlações com as respostas de coping (RSQ-D-Ped) foi a de "Ansiedade/Depressão" ("Quando penso que vou ficar só, em casa, fico assustado"; "É mais complicado ir passear por causa do diabetes", por exemplo). Esta subescala foi associada também com a idade, de forma que os adolescentes mais velhos apresentaram maiores níveis de estresse relacionados à ansiedade e depressão. Além disso, perceber-se ansioso ou deprimido por estar com diabetes é uma fonte de estresse relacionada a um maior Desengajamento voluntário (com Pensamento Fantasioso e Excitação Emocional) e a um Engajamento Involuntário (com Excitação Emocional e Fuga). Da mesma forma, esses estressores emocionais associaram-se à menor frequência de EE adaptativas e de
Engajamento por Coping de Controle Secundário, e vice-versa (Tabela 2).

O estresse relacionado às "queixas somáticas" apresentou correlação com uma maior frequência de respostas mal adaptativas ao estresse, com Pensamentos Intrusivos e Engajamento Involuntário. Correlacionou-se também a uma menor frequência de coping adaptativo, relativo à Regulação Emocional, Engajamento por Coping de Controle Secundário (com Reestruturação Cognitiva e Aceitação). Por outro lado, o estresse relativo às queixas somáticas também se correlacionou positivamente a um coping mais adaptativo, pelo Engajamento por Coping de Controle Primário, no sentido de tentar lidar diretamente com a fonte estressora. Para o estresse relativo a uma das queixas do DM, a hipoglicemia, observou-se o mesmo padrão de coping mais adaptativo, com maior Engajamento por Coping de Controle Primário e Expressão emocional, mas com menor Reestruturação Cognitiva (Tabela 2).

$\mathrm{O}$ estresse vinculado à relação médico/paciente apresentou correlação positiva com o tempo do diagnóstico, ou seja, piorando com o tempo. Da mesma forma, o estresse relacionado ao "regime terapêutico" associou-se às respostas de estresse mal adaptativas (Evitação, Ruminação, Pensamentos Intrusivos e Fuga) e a menos coping adaptativo (Regulação Emocional). Constatou-se também que, quanto maior é o estresse nos contextos escolar e social ("Sinto-me inferior aos outros", por exemplo), maior a frequência de Pensamentos Intrusivos (Tabela 2). 
Tabela 2. Correlações significativas entre os estressores e coping do diabetes mellitus tipo 1 em adolescentes $(\mathrm{N}=10)$

\begin{tabular}{|c|c|c|c|c|c|c|c|c|}
\hline \multirow{2}{*}{\multicolumn{2}{|c|}{$\begin{array}{c}\text { Respostas ao estresse } \\
\text { (RSQ-D-Ped) } \\
\text { Fatores }(* r)\end{array}$}} & \multicolumn{7}{|c|}{ Estresse face ao Diabetes (QSD-R) } \\
\hline & & $\begin{array}{c}\text { Estresse em } \\
\text { contextos } \\
\text { escolares e } \\
\text { sociais }\end{array}$ & $\begin{array}{c}\text { Ansiedade/ } \\
\text { depressão }\end{array}$ & $\begin{array}{l}\text { Estresse face à } \\
\text { hipoglicemia }\end{array}$ & $\begin{array}{l}\text { Relação } \\
\text { médico/ } \\
\text { paciente }\end{array}$ & $\begin{array}{c}\text { Regime } \\
\text { terapêutico } \\
\text { (tratamento) }\end{array}$ & $\begin{array}{l}\text { QSD-R } \\
\text { total }\end{array}$ & \\
\hline \multicolumn{9}{|c|}{ Respostas voluntárias } \\
\hline \multirow{2}{*}{$\begin{array}{l}\text { Engajamento } \\
\text { por Coping de } \\
\text { Controle } \\
\text { Primário }\end{array}$} & $\begin{array}{l}\text { Expressão } \\
\text { emocional }(M)\end{array}$ & 0,55 & 0,44 & 0,04 & $0,75^{*}$ & 0,35 & 0,21 & 0,42 \\
\hline & $\begin{array}{l}\text { Regulação } \\
\text { emocional }(P r)\end{array}$ & $-0,80^{*}$ & $-0,62$ & $-0,23$ & $-0,15$ & $-0,22$ & $-0,72 *$ & $-0,57$ \\
\hline \multirow{2}{*}{$\begin{array}{l}\text { Engajamento } \\
\text { por Coping de } \\
\text { Controle Se- } \\
\text { cundário }\end{array}$} & $\begin{array}{l}\text { Reestruturação } \\
\text { cognitiva }(P r)\end{array}$ & $-0,69 *$ & $-0,27$ & $-0,50$ & $-0,72 *$ & 0,09 & $-0,24$ & $-0,33$ \\
\hline & Aceitação $(P r)$ & $-0,73^{*}$ & $-0,38$ & $-0,58$ & $-0,57$ & 0,11 & $-0,34$ & $-0,44$ \\
\hline \multirow[t]{2}{*}{$\begin{array}{l}\text { Desengajamen- } \\
\text { to }\end{array}$} & $\begin{array}{l}\text { Pensamento } \\
\text { fantasioso }(M)\end{array}$ & 0,62 & 0,26 & $0,66^{*}$ & 0,44 & $-0,24$ & 0,19 & 0,31 \\
\hline & Evitação $(P r)$ & 0,59 & 0,56 & 0,45 & 0,48 & $-0,02$ & $0,63^{*}$ & 0,60 \\
\hline \multicolumn{9}{|c|}{ Respostas involuntárias } \\
\hline \multirow[t]{3}{*}{$\begin{array}{l}\text { Engajamento } \\
\text { involuntário }\end{array}$} & $\begin{array}{l}\text { Pensamentos } \\
\text { intrusivos }(P r)\end{array}$ & $0,74 *$ & $0,77^{*}$ & 0,17 & 0,12 & 0,28 & $0,66^{*}$ & $0,66^{*}$ \\
\hline & $\begin{array}{l}\text { Excitação emo- } \\
\text { cional }(P r)\end{array}$ & 0,57 & 0,39 & $0,65^{*}$ & 0,36 & $-0,34$ & 0,44 & 0,44 \\
\hline & $\begin{array}{l}\text { Ruminação } \\
(P r)\end{array}$ & 0,16 & 0,52 & 0,51 & $-0,09$ & 0,07 & $0,63^{*}$ & 0,57 \\
\hline \multirow{2}{*}{$\begin{array}{l}\text { Desengajamen- } \\
\text { to involuntário }\end{array}$} & Fuga $(M)$ & 0,54 & 0,52 & $0,70^{*}$ & 0,23 & 0,02 & 0,41 & 0,53 \\
\hline & Fuga $(P r)$ & 0,44 & 0,58 & $0,72 *$ & 0,11 & $-0,06$ & $0,72 *$ & $0,63^{*}$ \\
\hline
\end{tabular}

Nota: *r significativo pelo teste de correlação de Spearman, ao nível de $p \leq 0,05 ; M=$ Média; $P r=$ média proporcional; Coping de controle primário: ações que visam a alterar a situação estressora ou da emoção por ela eliciada; Coping de controle secundário: respostas de adaptação ao problema; Reestruturação cognitiva: processo de reconceitualização de uma ideia ou atitude, vendo-a de uma perspectiva diferente; Ruminação: padrão de pensamentos persistentes, com foco em desfechos e emoções negativos; Pensamentos intrusivos: eventos mentais que interrompem o curso da ação, a despeito dos esforços persistentes para evitá-los; Interferência cognitiva: bloqueio da aprendizagem ou memória pelo aprendizado ou recordação de outro material conflitante.

Os resultados significativos encontrados entre os comportamentos de autocuidado (QAD) e o coping do diabetes (RSQ-D-Ped) indicaram que, quanto maior o Engajamento por Coping de Controle Secundário, com respostas de Aceitação. Quanto menor a média proporcional de Pensamento Fantasioso e de Entorpecimento Emocional, maior é a adesão aos cuidados com a "alimentação geral", e vice-versa (Tabela 3). 
Tabela 3. Correlações entre coping do diabetes mellitus tipo 1 e comportamentos de autocuidado em adolescentes $(\mathrm{N}=10)$

\begin{tabular}{|c|c|c|c|c|c|c|c|c|}
\hline \multirow{2}{*}{\multicolumn{2}{|c|}{$\begin{array}{c}\text { Respostas ao estresse } \\
\text { (RSQ-D-Ped) } \\
\text { Fatores }(* r) \\
\text { Alimentação Geral } \\
\left({ }^{*}\right)\end{array}$}} & \multicolumn{7}{|c|}{ Atividades de Autocuidado no diabetes (QAD) } \\
\hline & & $\begin{array}{c}\text { Alimentação } \\
\text { Específica } \\
\left(*^{*} \mathrm{r}\right)\end{array}$ & $\begin{array}{l}\text { Atividade } \\
\text { Física } \\
\left(*^{r}\right)\end{array}$ & $\begin{array}{l}\text { Monitora- } \\
\text { mento de } \\
\text { glicemia } \\
\quad\left({ }^{*} r\right)\end{array}$ & $\begin{array}{l}\text { Cuidado com } \\
\text { os pés } \\
(* r)\end{array}$ & $\begin{array}{l}\text { Medicação } \\
\left({ }^{*} r\right)\end{array}$ & $\begin{array}{c}\text { QAD } \\
\text { total } \\
(* r)\end{array}$ & \\
\hline \multicolumn{9}{|c|}{ Respostas voluntárias } \\
\hline $\begin{array}{l}\text { Engajamento por } \\
\text { Coping de Con- } \\
\text { trole Primário }\end{array}$ & Escore $(P r)$ & 0,01 & 0,29 & $0,74^{*}$ & $-0,53$ & 0,25 & $-0,48$ & 0,16 \\
\hline \multirow{3}{*}{$\begin{array}{l}\text { Engajamento por } \\
\text { Coping de Con- } \\
\text { trole Secundário }\end{array}$} & Escore $(P r)$ & 0,39 & $-0,09$ & 0,38 & $-0,35$ & $0,67 *$ & $-0,18$ & 0,48 \\
\hline & Aceitação $(M)$ & $0,79^{*}$ & $-0,21$ & 0,22 & $-0,05$ & 0,32 & 0,29 & 0,53 \\
\hline & Distração $(P r)$ & 0,15 & $-0,01$ & 0,53 & $-0,21$ & $0,64 *$ & $-0,38$ & 0,17 \\
\hline Desengajamento & $\begin{array}{l}\text { Pensamento } \\
\text { fantasioso }(P r)\end{array}$ & $-0,67 *$ & 0,26 & $-0,12$ & 0,14 & $-0,29$ & $-0,21$ & $-0,32$ \\
\hline \multicolumn{9}{|c|}{ Respostas involuntárias } \\
\hline \multirow[t]{3}{*}{$\begin{array}{l}\text { Engajamento } \\
\text { involuntário }\end{array}$} & $\begin{array}{l}\text { Pensamentos } \\
\text { intrusivos }(P r)\end{array}$ & $-0,37$ & $-0,41$ & $-0,52$ & 0,04 & $-0,25$ & 0,01 & $-0,70$ \\
\hline & $\begin{array}{l}\text { Excitação fisio- } \\
\text { lógica }(P r)\end{array}$ & $-0,06$ & 0,17 & $0,64 *$ & $-0,28$ & 0,16 & $-0,50$ & 0,09 \\
\hline & Ruminação $(P r)$ & 0,09 & $-0,29$ & $-0,79 *$ & $0,70^{*}$ & $-0,50$ & $0,72 *$ & $-0,10$ \\
\hline $\begin{array}{l}\text { Desengajamento } \\
\text { involuntário }\end{array}$ & $\begin{array}{l}\text { Entorpecimento } \\
\text { emocional }(P r)\end{array}$ & $-0,77 *$ & 0,03 & $-0,03$ & 0,35 & 0,01 & $-0,62$ & $-0,45$ \\
\hline
\end{tabular}

Nota: ${ }^{*} p \leq 0,05$ a partir do Teste de Correlação de Spearman; $P r=$ escore proporcional; $M=$ média; Coping de controle primário: ações que visam a alterar a situação estressora ou da emoção por ela eliciada; Coping de controle secundário: respostas de adaptação ao problema; Ruminação: padrão de pensamentos persistentes, com foco em desfechos e emoções negativos; Pensamentos intrusivos: eventos mentais que interrompem o curso da ação, a despeito dos esforços persistentes para evitá-los.

O escore geral do QAD (comportamentos de autocuidados) e do COPE-DIA (coping adaptativo e mal adaptativo do diabetes) não apresentaram correlações significativas. Contudo, houve correlações positivas entre a monitorização da glicemia e apresentar afeto negativo (tristeza e medo), e também com uma maior percepção de ameaça à NPB de Autonomia. Fazer "Atividade Física" associou-se com menos medo e mais Autoconfiança. Ter que usar a medicação também apresentou correlação positiva com a tristeza e a percepção de ameaça à NPB de Autonomia. Os adolescentes se identificaram especialmente com a S2 (adesão), que inclui o monitoramento da glicemia (Tabela 4). 
Tabela 4. Correlações entre as respostas emocionais, coping e comportamentos de autocuidado em adolescentes com diabetes mellitus tipo $1(\mathrm{~N}=10)$

\begin{tabular}{|c|c|c|c|c|c|}
\hline \multirow[b]{2}{*}{$\begin{array}{l}\text { Comportamen- } \\
\text { tos } \\
\text { de Autocuida- } \\
\text { dos } \\
\text { (QAD) }\left({ }^{*} r\right)\end{array}$} & \multicolumn{5}{|c|}{$\begin{array}{l}\text { Reações emocionais e coping do Diabetes } \\
\text { (COPE-DIA) }\end{array}$} \\
\hline & 胥 & 异 & 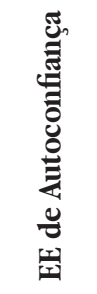 & 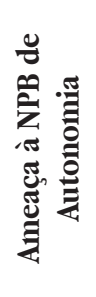 & 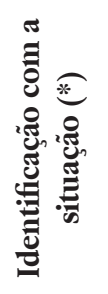 \\
\hline $\begin{array}{l}\text { Alimentação } \\
\text { geral }\end{array}$ & 0,29 & $-0,01$ & $-0,14$ & 0,07 & $-0,16$ \\
\hline $\begin{array}{l}\text { Alimentação } \\
\text { específica }\end{array}$ & $-0,48$ & $-0,42$ & 0,41 & $-0,34$ & $-0,50$ \\
\hline Atividade física & $-0,43$ & $-0,63$ & $0,63^{*}$ & $-0,59$ & $-0,45$ \\
\hline $\begin{array}{l}\text { Monitoramento } \\
\text { de glicemia }\end{array}$ & $0,78^{*}$ & $0,64^{*}$ & $-0,29$ & $0,88^{*}$ & $0,77^{*}$ \\
\hline $\begin{array}{l}\text { Cuidado com } \\
\text { os pés }\end{array}$ & $-0,18$ & $-0,48$ & 0,15 & $-0,34$ & $-0,26$ \\
\hline Medicação & $0,68^{*}$ & 0,58 & $-0,52$ & $0,73^{*}$ & 0,44 \\
\hline QAD total & 0,00 & $-0,24$ & 0,14 & 0,08 & $-0,12$ \\
\hline
\end{tabular}

Nota: EE: estratégias de enfrentamento; NPB: necessidade psicológica básica; (*) COPE-DIA: propõe três situações estressoras para adolescentes responder como lidariam.

O Quadro 1 apresenta uma síntese das correlações entre os estressores, o coping e os comportamentos de autocuidado. Para o principal estressor, relacionado à hipoglicemia, as respostas de coping foram de Controle Primário, mas com menos reestruturação cognitiva. No geral, os estressores ameaçam a NPB de Competência. Os adolescentes lidam com o regime terapêutico de forma mal adaptativa, diminuindo a adesão às atividades físicas. Os estressores relacionados à ansiedade/depressão apresentaram maior número de correlações, especialmente com respostas de estresse involuntárias e de desengajamento da situação.
Quadro 1. Resumo das correlações entre os estressores, o coping e os comportamentos de autocuidados em adolescentes com diabetes mellitus tipo $1(\mathrm{~N}=10)$

\begin{tabular}{|c|c|c|}
\hline $\begin{array}{l}\text { Estressores do } \\
\text { diabetes }\end{array}$ & $\begin{array}{c}\text { Respostas de estresse } \\
\text { involuntárias e coping } \\
\text { (RSQ-D-Ped) }\end{array}$ & $\begin{array}{c}\text { Adesão } \\
\text { (QAD) }\end{array}$ \\
\hline $\begin{array}{l}\text { Estresse face à } \\
\text { Hipoglicemia } \\
\text { (QSD-R) }\end{array}$ & $\begin{array}{l}\text { (+) Coping de Controle Primário } \\
\text { (+) Expressão Emocional } \\
\text { (-) Reestruturação Cognitiva }\end{array}$ & - \\
\hline $\begin{array}{l}\text { Regime Terapêutico } \\
\text { (Tratamento) (QS- } \\
\text { D-R) }\end{array}$ & $\begin{array}{l}\text { (-) Regulação Emocional } \\
\text { (+) Evitação } \\
\text { (+) Pensamentos Intrusivos (inv.) } \\
\text { (+) Ruminação (inv.) } \\
\text { (+) Fuga (inv.) }\end{array}$ & $\begin{array}{l}\text { (-) Atividade } \\
\text { Física }\end{array}$ \\
\hline $\begin{array}{l}\text { Queixas Somáticas } \\
\text { (QSD-R) }\end{array}$ & $\begin{array}{l}\text { (+) Coping de Controle Primário } \\
\text { (-) Regulação Emocional } \\
\text { (-) Coping de Controle Secundário } \\
\text { (-) Aceitação } \\
\text { (+) Engajamento Involuntário } \\
\text { (+) Pensamento Intrusivo (inv.) }\end{array}$ & - \\
\hline $\begin{array}{l}\text { Estresse em con- } \\
\text { textos escolares e } \\
\text { sociais (QSD-R) }\end{array}$ & (+) Pensamento Intrusivo (inv.) & - \\
\hline $\begin{array}{l}\text { Ansiedade/ } \\
\text { Depressão } \\
\text { (QSD-R) }\end{array}$ & $\begin{array}{l}\text { (+) Idade } \\
\text { (-) Coping de Controle Secundário } \\
\text { (+) Desengajamento } \\
\text { (+) Pensamento Fantasioso } \\
\text { (+) Engajamento Involuntário } \\
\text { (+) Excitação Emocional (inv.) } \\
\text { (+) Fuga (inv.) }\end{array}$ & - \\
\hline $\begin{array}{l}\text { Relação Médico/ } \\
\text { Paciente (QSD-R) }\end{array}$ & (+) Tempo de diagnóstico & - \\
\hline QSD-R Total & $\begin{array}{l}\text { (+) Engajamento Involuntário } \\
\text { (+) Pensamento Intrusivo (inv.) } \\
\text { (+) Fuga (inv.) }\end{array}$ & - \\
\hline $\begin{array}{l}\text { Média dos Estresso- } \\
\text { res (RSQ-D-Ped) }\end{array}$ & $\begin{array}{l}\text { (+) NPB de Competência } \\
\text { (COPE-DIA) }\end{array}$ & - \\
\hline
\end{tabular}

Nota: NPB: necessidade psicológica básica; Inv.: resposta involuntária de estresse; Coping de controle primário: ações que visam a alterar a situação estressora ou da emoção por ela eliciada; Coping de controle secundário: respostas de adaptação ao problema; Reestruturação cognitiva: processo de reconceitualização de uma ideia ou atitude, vendo-a de uma perspectiva diferente; Ruminação: padrão de pensamentos persistentes, com foco em desfechos e emoções negativos; Pensamentos intrusivos: eventos mentais que interrompem o curso da ação, a despeito dos esforços persistentes para evitá-los; Interferência cognitiva: bloqueio da aprendizagem ou memória pelo aprendizado ou recordação de outro material conflitante.

\section{DISCUSSÃO}

Metade dos 10 adolescentes desta amostra apresentou baixa adesão aos comportamentos de autocuidado indicados pelos profissionais de saúde, confirmando dados da literatura ${ }^{24}$. Colaboram para a não adesão ao 
tratamento a baixa escolaridade e a falta de conhecimento no manejo da doença, tornando-se fatores importantes em virtude da complexidade terapêutica, o que também dificulta a abordagem dos profissionais ${ }^{2}$. Esta constatação é coerente com os dados encontrados neste estudo, em que se observou uma baixa escolaridade das mães. Assim, fatores familiares e as questões econômicas, além das características psicológicas, podem influenciar o controle glicêmico ${ }^{8}$.

Lidar com a doença de forma mais adaptativa, com EE de Aceitação, relacionou-se com a adesão ao tratamento, por meio da alimentação; ou seja, aceitando a situação, os adolescentes se alimentam de forma mais adequada. Esse resultado é coerente com a pesquisa realizada por alguns autores ${ }^{8,10,23}$, em que os adolescentes que demonstraram maior aceitação da doença apresentaram uma melhor adaptação ao regime de tratamento.

Para esses adolescentes, o principal estressor relacionado ao cuidado do diabetes foi a condição de sofrer com a hipoglicemia, o que pode ter relações com a maior adesão ao monitoramento de glicemia. O sentimento de culpa em relação ao não cumprimento da dieta e das orientações feitas pela equipe de saúde, associado à cobrança dos pais, acabam por gerar ansiedade ou depressão, principalmente naqueles adolescentes mais velhos, possivelmente por buscarem maior autonomia e assumirem maior responsabilidade pelo seu tratamento. O regime terapêutico e as queixas somáticas também se destacaram como estressores nesta amostra. Neste estudo, os adolescentes buscaram o suporte para a expressão e a regulação emocional, bem como para a solução de problemas, principalmente, nos pais, em Deus, irmãos e nos amigos. Esses resultados são positivos, pois o suporte social é apontado como um fator de proteção, contribuindo principalmente para o controle metabólico, manejo da doença e adesão ao tratamento ${ }^{7-8,26}$.

As relações entre estressores da doença e seu enfrentamento também foram identificadas na relação entre os estressores vinculados ao regime terapêutico e ter que fazer atividade física. É comprovado que fazer exercícios físicos auxilia no controle do DM1 ${ }^{27}$. Frente a esses estressores, os adolescentes relataram mais respostas involuntárias de estresse, como a evitação, pensamentos intrusivos, ruminação e fuga. Assim, quanto mais estressores relacionados ao tratamento, maior é a frequência desse coping mal adaptativo e menor é a adesão à atividade física. Diante desse quadro reativo involuntário, menor é a regulação emocional, que seria uma forma de resposta voluntária para lidar diretamente com o estressor, associando-se também a uma menor a adesão à prática de atividade física. Essas respostas de coping também foram encontradas em outros estudos com adolescentes com DM1, aplicando o mesmo instrumento (RSQ-D-Ped) ${ }^{8 \cdot 10}$.

Diferentes EE podem ser acionadas caso o estressor seja avaliado ou percebido como uma ameaça ou um desafio ${ }^{15,28}$. Isso ocorreu com todos os adolescentes, que apresentaram tanto EE adaptativas, quanto mal adaptativas. Além disso, a avaliação de três situações específicas, feita pelo COPE-DIA, com os principais estressores da doença, demonstrou que muitos adolescentes as avaliaram como uma ameaça. Quando analisada a orientação das respostas de coping, em termos de querer sair da situação ou fugir, a maioria dos adolescentes, nas três situações, relatou querer se afastar. Em relação à NPB de Autonomia, os adolescentes sentiram-se ameaçados na situação S2-Adesão ao tratamento e na S3-Relacionamento com os pais. Na S1-Estresse social, observou-se baixa percepção de ameaça à NPB de Autonomia. Esses dados mostram que, de acordo com cada situação estressora da doença, o adolescente, ora tem percepção de ameaça, ora de desafio, alterando assim os comportamentos de coping apresentados.

Os adolescentes relataram que lidariam com as três situações estressoras, apresentando, principalmente, EE relativas às famílias de coping de Isolamento, Fuga, Busca de Suporte e Solução de Problemas. Os dados evidenciam que o suporte recebido por esses adolescentes, principalmente através do apoio dos pais, da ajuda para a satisfação de sua NPB de Competência, oferecendo apoio e auxiliando na solução de problemas, contribui para melhorar o processo de adesão ao tratamento. Os adolescentes desejam autonomia, ao mesmo tempo em que desejam e precisam ser cuidados. O suporte da família na adolescência nesse processo é fundamental para a promoção da autonomia e também satisfazer a necessidade de relacionamento, evitando, assim, a ocorrência do isolamento como estratégia de enfrentamento da situação por parte do adolescente ${ }^{13}$. 
A reação emocional de raiva foi a mais frequente nas três situações avaliadas pelo COPE-DIA, sendo mais intensa na S2-Adesão ao tratamento e na S3-Relacionamento com os pais. Nas pessoas com diabetes, as reações emocionais são um aspecto importante e apresentam maiores variações, devido aos sentimentos de perda e frustração associados. Na adversidade, emergem inúmeras emoções, que serão positivas ou negativas de acordo com o processo de engajamento ou distanciamento do evento estressor. Essas emoções interpelam o indivíduo, e tal como foi expresso por Skinner et al. ${ }^{13}$, as emoções são um domínio integrante e permanente do processo de coping, que indica a regulação emocional, além de regulação do comportamento, da cognição e da motivação.

\section{CONCLUSÃO}

O presente estudo buscou uma relação inovadora nos estudos da área no país - estresse, coping e adesão ao tratamento - pensando na patologia específica, DM1, e no coping compreendido como um processo desenvolvimentista, tendo em vista que apresenta características específicas de acordo com a faixa etária, no caso deste estudo, os adolescentes. A aplicação da MTC nesse contexto de saúde mostrou-se útil para compreender melhor o processo de estresse e seu enfrentamento em adolescentes, oferecendo dados que podem subsidiar intervenções mais eficazes no tratamento do diabetes.

Considerando a alta frequência de estressores ligados ao relacionamento com os pais e a importância atribuída ao suporte social pelos adolescentes, sugere-se incluir, em estudos futuros, essas variáveis para entender o processo de adesão ao tratamento pelo adolescente. Compreender a dinâmica familiar, os relacionamentos estabelecidos nesse contexto e os estilos parentais são de fundamental importância para analisar o suporte social e a possibilidade de construção da autonomia. Pode-se, assim, contribuir no processo do jovem assumir a responsabilidade pelo seu tratamento, aderindo, dessa forma, às orientações dos profissionais da saúde na execução dos comportamentos de autocuidado.

Os dados obtidos sobre a maior frequência de respostas involuntárias de estresse e de desengajamento desses adolescentes com DM1 indicam a importância dos profissionais de saúde prevenirem esses comportamentos nessa população. A promoção de um coping mais adaptativo, de controle primário e secundário, pode melhorar a adesão ao tratamento e a qualidade de vida.

Outro desdobramento deste estudo poderia incluir o questionamento sobre os motivos da resistência à adesão aos comportamentos de autocuidado do diabetes. Para isso, é preciso uma metodologia de estudo capaz de registrar dados não apenas da frequência dos comportamentos, mas dos motivos pelos quais estes comportamentos são ou não realizados. Outro aspecto que merece investigação é o ambiente escolar e o preparo de professores e funcionários para facilitar a adaptação do adolescente à sua condição de doença crônica. O espaço acadêmico também é responsável para o bom desenvolvimento social do adolescente com seus pares e, neste caso, um adolescente, que, além de enfrentar os desafios impostos pela fase de desenvolvimento, tem o agravante de ser portador de uma doença crônica.

Apesar do tamanho amostral limitar a extrapolação dos resultados, este trabalho apresenta uma proposta metodológica e uma abordagem teórica mais profícua para estudos com essa população. Os dados encontrados fornecem subsídios que contribuem para o entendimento da maneira como os adolescentes percebem sua doença e a forma como enfrentam o tratamento. $O$ conhecimento desses fatores condicionantes da adesão ao tratamento do DM1 é fundamental para o bem-estar dos adolescentes e para melhoria de sua qualidade de vida. Espera-se que, com intervenções adequadas, os adolescentes consigam assumir a responsabilidade do seu tratamento, tornando-se independentes e, diminuindo, assim, a dificuldade em aderir aos comportamentos de autocuidado.

\section{AGRADECIMENTOS}

À Coordenação de Aperfeiçoamento de Pessoal de Nível Superior (CAPES), pela concessão de bolsa de doutorado à primeira autora; ao Conselho Nacional de Desenvolvimento Científico e Tecnológico (CNPq), pela concessão de bolsa de produtividade em pesquisa para a última autora, orientadora do estudo - auxílio à pesquisa no 455543/2014-3; à Direção da instituição onde foi realizada a coleta de dados. 


\section{REFERÊNCIAS}

1. WHO (World Health Organization). 2016. Global report on diabetes. World Health Organization. France. Disponível de: http://apps.who.int/iris/ bitstream/handle/10665/204871/9789241565257_ eng.pdf; jessionid = C 1 F CA2 DEC 18 D BE413E0C9E4B9239E3D2? sequence $=1$

2. Sociedade Brasileira de Diabetes [SBD] (2017). Diretrizes da Sociedade Brasileira de Diabetes. Disponível de: https://www.diabetes.org.br/profissionais/images/2017/diretrizes/diretrizes-sbd-2017-2018.pdf

3. Levesque C. Management of diabetes in children and adolescents. Nurs. Clin. North America. 2017; 52(4): 589-603.

4. Compas BE, Jaser SS, Bettis AH, Watson KH, Gruhn MA, Dunbar JP, et al. Coping, emotion regulation, and psychopathology in childhood and adolescence: A meta-analysis and narrative review. Psychol. bull. 2017;143(9):939-991.

5. Varni JW, Delamater AM, Hood KK, Raymond JK, Driscoll KA, Wong JC, et al. Diabetes symptoms predictors of health-related quality of life in adolescents and young adults with type 1 or type 2 diabetes. Qual. Life Res. 2018;1-9.

6. Driscoll KA, Raymond J, Naranjo D, Patton SR. Fear of hypoglycemia in children and adolescents and their parents with type 1 diabetes. Curr. diab. rep. 2016; 16(8):77.

7. Cassarino-Perez L, Alves CF, Dell'Aglio DD Suporte social em adolescentes com Diabete Mellitus Tipo I: Uma revisão sistemática. Rev. SPAGESP. 2014; 15(1): 33-48.

8. Jaser SS, Faulkner M, Whittemore R, Jeon S, Murphy $\mathrm{K}$, Delamater A, et al. Coping, self-management, and adaptation in adolescents with Type 1 Diabetes. Ann Behav Med. 2012;43(3):311-319.

9. Jaser SS, Patel N, Xu M, Tamborlane WV, Grey M. Stress and coping predicts adjustment and glycemic control in adolescents with Type 1 Diabetes. Ann Behav Med. 2017; 51(1):30-38.

10. Jaser SS, White LE (2011). Coping and resilience in adolescents with type 1 diabetes. Child Care Health Dev. 2011; 37(3):335-42.
11. Thorpe CT, Fahey LE, Johnson H, Deshpande M, Thorpe JM, Fisher EB. Facilitating healthy coping in patients with diabetes: a systematic review. Diabetes Educ. 2013;39(1):33-52.

12. Compas BE, Connor-Smith JK, Saltzman H, Thomsen AH, Wadsworth M E. Coping with stress during childhood and adolescence: Progress, problems, and potential in theory and research. Psychol. Bull. 2001; 127(1):87-127.

13. Skinner EA, Zimmer-Gembeck MJ. The development of coping: Stress, neurophysiology, social relationships, and resilience during childhood and adolescence. USA: Springer. 2016.

14. Ramos FP, Enumo SRF, Paula KMP. Teoria Motivacional do Coping: Uma proposta desenvolvimentista de análise do enfrentamento do estresse. Estud. Psicol. (Campinas). 2015;32(2):269-79.

15. Skinner EA, Edge K, Altman J, Sherwood H. Searching for the structure of coping: A review and critique of category systems for classifying ways of coping. Psychol. Bull. 2003;129(2):216-69.

16. Skinner EA, Welborn JG. Coping during childhood and adolescence: A motivational perspective. In D. L. Featherman, R. M. Lerner, \& M. Perlmutter (Eds.), Life-Span Development and Behavior (v. 12). Hillsdale, NJ: Lawrence Erlbaum Associates. 1994. p. 91-133.

17. Associação Brasileira de Empresas de Pesquisa [ABEP]. Critério de Classificação Econômica Brasil. 2013. Disponível em: http://www.abep.org. Acesso em: 10 de janeiro de 2014.

18. Toobert DJ, Hampson SE, Glasgow RE. The Summary of Diabetes Self-Care Activities Measure: Results from 7 studies and a revised scale. Diabetes Care. 2000;23 (7):943-950.

19. Michaels MJ, Coral MHS, Sakae TM, Damas TB, Furlanetto LM. Questionário de Atividades de Autocuidado com o Diabetes: Tradução, adaptação e avaliação das propriedades psicométricas. Arq. Bras. Endocrinol. Metab. 2010;54(7):644-51.

20. Herschback P, Duran G, Waadt S, Zetler A, Amm C, Marten-Mittag. Psychometric properties of the questionnaire on stress in patients with diabetes-revised (QSD-R). Health Psychol. 1997;6:171-4. 
21. Almeida JP, Pereira MG. Propriedades psicométricas do questionário de stress face à diabetes em adolescentes portugueses. Psicol. Saúde Doenças. 2012;13(2):356-71.

22. Connor-Smith JK, Compas BE, Wadsworth ME, Thomsen AH, Saltzman H. Responses to stress in adolescence: Measurement of coping and involuntary stress responses. J. Consult. Clin. Psychol. 2000;68 (6):976-92.

23. Lees DC. An empirical investigation of the motivational theory of coping in middle to late childhood. Doctoral Thesis of Philosophy in Clinical Psychology, Griffith University, QLD, Australia 2007.

24. Murphy J, Rayman G, Skinner TC. Psycho-educational interventions for children and young people with type 1 diabetes. Diabet. Med 2006;23(9):935-943.

25. Chilton R, Pires-Yfantouda R Understanding adolescent type 1 diabetes self-management as an adaptive process: A grounded theory approach. Psychol. Health. 2015;30(12):1486-504.

26. Kamody RC, Berlin KS, Rybak TM, Klages KL, Banks GG, Ali JS, et al. Psychological flexibility among youth with type 1 diabetes: Relating patterns of acceptance, adherence, and stress to adaptation. Behav Med. $2017 ; 1-9$.

27. D'Angelo FA, Leatte EP, Defani MA. O exercício físico como coadjuvante no tratamento do Diabetes. Saúde e Pesquisa, 2015;8(1):157-66.

28. Modecki KL, Zimmer-Gembeck MJ, Guerra N. Emotion regulation, coping, and decision making: three linked skills for preventing externalizing problems in adolescence. Child. Dev. 2017;88(2):417-26.

Recebido em: 23/08/2018

Aceito em: 28/12/2018 